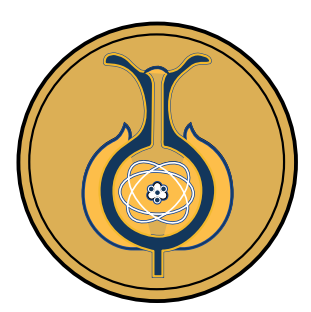

\title{
Evaluación del impacto ambiental en una industria gráfica, que utiliza impresión litográfica tipo "offset"
}

\author{
Environmental impact assessment of a graphic company that uses offset \\ lithographic printing
}
Avaliação do impacto ambiental em uma indústria gráfica, que utiliza a impressão litográfica tipo offset

\section{Wendy Villalobos-González ${ }^{1}$ José Pablo Sibaja-Brenes ${ }^{2}$ José Carlos Mora-Barrantes ${ }^{2} \bullet$ Benjamín Álvarez-Garay ${ }^{1}$}

Received: Jan/13/2020 • Accepted: Aug/31/2020 • Published: Jan/31/2021

\section{Resumen}

La industria gráfica se dedica a la impresión de documentos, que utilizan diferentes tecnologías, una de estas es la impresión litográfica tipo "offset". El proceso productivo de este tipo de industria, así como el uso de sustancias químicas, generan impactos ambientales que pueden afectar de forma negativa al ambiente. Una evaluación de impacto ambiental (EIA), tiene la finalidad de identificar y evaluar los impactos ambientales y generar medidas preventivas, correctivas y compensatorias, en pro del ambiente. Entre los principales impactos teóricos de este tipo de industria están: generación de aguas residuales, emisión de gases, generación de ruido y residuos sólidos. En el presente proyecto de investigación se realizó una identificación, valorización y priorización de los principales aspectos ambientales (AAs) e impactos ambientales (IAs) generados en una industria litográfica tipo "offset" en Costa Rica. Con el objetivo de conocer la situación ambiental de la industria, se realizaron análisis fisicoquímicos de aguas residuales (AR), compuestos orgánicos volátiles (VOCs), partículas totales en suspensión (PTS), ruido y luminosidad. La identificación de los AAs se efectuó en conjunto con los funcionarios de la empresa, con expertos en gestión ambiental y mediante sesiones de trabajo con grupos focales, mediante herramientas como: revisión bibliográfica, lista de chequeo, entrevistas e inspecciones de campo. La valorización y priorización de los AAs identificados se llevó a cabo mediante la metodología denominada Matriz de Importancia de Impacto Ambiental (MIIA). En total se identificaron 16 AAs y 5 IAs. El AA crítico más negativo para el ambiente es la emisión de VOCs, lo cual se debe principalmente a las altas concentraciones encontradas en el ambiente interno de la planta de producción y a la ineficiencia de los sistemas de extracción general o localizada. Los tres principales IAs de carácter negativo al ambiente encontrados fueron: contaminación de la atmósfera, afectación a la salud y agotamiento de recursos no renovables, con valores de impacto ambiental de $-64,-62$ y -62, respectivamente.

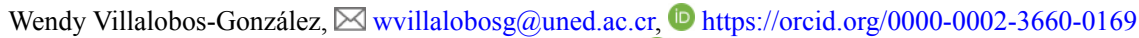
José Pablo Sibaja-Brenes, \jose.sibaja.brenes@una.cr, (D https://orcid.org/0000-0002-7056-2717 José Carlos Mora-Barrantes, $\triangle$ jose.mora.barrantes@una.cr, (D) https://orcid.org/0000-0002-0409-5276 Benjamín Álvarez-Garay, \balvarez@uned.ac.cr, (D https://orcid.org/0000-0002-2180-0870

1 Escuela de Ciencias Exactas y Naturales, Universidad Estatal a Distancia, San José, Costa Rica.

2 Escuela de Química, Universidad Nacional, Heredia, Costa Rica.
} 
Palabras clave: industria gráfica; evaluación ambiental; aspecto e impacto ambiental; residuos emisiones

\section{Abstract}

The graphic industry is dedicated to printing documents using different technologies, one of which being offset lithographic printing. This production process and the use of chemical substances generate a negative environmental impact. The purpose of this environmental impact assessment (EIA) is to identify and evaluate the environmental impact and generate preventive, corrective, and compensatory measures. Generally, the main effects of this type of industry include generation of wastewater, gas emissions, noise, and solid waste. This research identified, assessed, and prioritized the main environmental aspects (AAs) and environmental impacts (IAs) generated in the offset lithographic industry in Costa Rica. Physicochemical analyses of wastewater (AR), volatile organic compounds (VOCs), total suspended particles (TSPs), noise, and luminosity were conducted to know the environmental situation of the industry. Environmental aspects were identified in conjunction with company officials, environmental management experts and through work sessions with focus groups, using tools such as bibliographic reviews, checklists, interviews and field inspections. The environmental aspects identified were assessed and prioritized using the Matrix of Environmental Impact Importance (MIIA) methodology. A total of 16 environmental aspects and 5 environmental impacts were identified. The most critical negative environmental aspect is VOC emissions, mainly due to the high concentrations found in the internal production plant and inefficient general or localized extraction systems. The three main negative environmental impacts were air pollution, health effects, and depletion of non-renewable resources, with environmental impact values of $-64,-62$ and -62 , respectively.

Keywords: graphic industry; environmental assessment; environmental aspect and impact; waste emissions

\section{Resumo}

A indústria gráfica dedica-se à impressão de documentos por meio de diferentes tecnologias, sendo uma delas a impressão litográfica tipo offset. 0 processo produtivo, assim como 0 uso de substâncias químicas, gera impacto ambiental (EIA) negativo. Uma avaliação disso tem a finalidade de identificar, avaliar e gerar medidas preventivas, corretivas e compensatórias. Geralmente, os principais efeitos deste tipo de indústria são: emissão de gases e geração de águas residuais, ruídos e resíduos sólidos. No presente projeto de pesquisa foi realizada uma identificação, valorização e priorização dos principais aspectos ambientais (AAs) e impactos ambientais (IAs) gerados em uma indústria litográfica tipo offset na Costa Rica. Com o objetivo de conhecer a situação ambiental da indústria, foram realizadas análises físico-químicas de águas residuais (AR), compostos orgânicos voláteis (VOCs), partículas totais em suspensão (PTS), ruído e luminosidade. A identificação dos AAs foi realizada em conjunto com os funcionários da empresa, com especialistas em gestão ambiental e mediante sessões de trabalho com grupos focais, utilizando ferramentas como: revisão bibliográfica, lista de controle, entrevistas e inspeções de campo. A valorização e priorização dos AAs identificados foram realizadas por meio da metodologia denominada Matriz de Importância de Impacto Ambiental (MIIA). No total, foram identificados 16 AAs e 5 IAs. 0 AA crítico mais negativo para 0 ambiente é a emissão de VOCs, resultada, principalmente, das altas concentrações encontradas no ambiente interno da planta de produção e à ineficiência dos sistemas de extração geral ou localizada. Os três principais IAs de carácter negativo ao meio ambiente encontrados foram: contaminação da atmosfera, impacto na saúde e esgotamento de recursos não renováveis, com valores de impacto ambiental de $-64,-62$ e -62 , respectivamente.

Palavras-chave: indústria gráfica; avaliação ambiental; aspecto e impacto ambiental; resíduos emissões. 


\section{INTRODUCCIÓN}

La industria de las artes gráficas y la publicación de documentos impresos fueron desarrolladas en el siglo XI con la invención de la imprenta china (Richardson, 1998), con los años ha experimentado un crecimiento y evolución, que amplía la tecnología utilizada (Medaglia y Vargas, 2013). La técnica más utilizada es la litografía la cual conlleva impresión "offset. En este tipo de impresión se genera una plancha con la información de la impresión final, la cual es revelada en una máquina denominada control directo a plancha (CTP) (García, Leone y Williams, 2014).

Un proceso de impresión tradicional CTP generalmente se divide en tres etapas: pre-impresión, impresión y post-impresión. En la pre-impresión se incluye las actividades de diseño, diagramación y edición, transfiriéndose el diseño a un portador de imágenes (plancha). Esta operación involucra procesos físicos o químicos tales como la exposición a luz ultravioleta (UV) o láser, fotograbado, revelado y procesamiento posterior. En la impresión, la plancha se coloca en la impresora. Por último, en la post-impresión se incluyen los acabados finales de los materiales para comercialización (Occupational Safety and Health Branch Labour Department, 2004). Entre las materias primas utilizadas sobresalen: papel, tintas, planchas, agua y productos químicos (García, Leone y Williams, 2014; Occupational Safety and Health Branch Labour Department, 2004).

Resultado del proceso productivo de la industria litográfica se generan aguas residuales, emisiones gaseosas y residuos sólidos ordinarios y de carácter especial (García, Leone y Williams, 2014; Dimate y Chirley, 2014). Las aguas residuales (AR) provenientes de la industria gráfica son muy variables en composición química, debido a la diversidad de productos que utilizan en los procesos. En general, se caracterizan por tener altas demandas químicas de oxígeno (DQO), permanencia de color, sólidos suspendidos totales (SST) (Prica et al 2016); lo que produce un índice de biodegradabilidad bajo $\left(\mathrm{DBO}_{5} / \mathrm{DQO}\right.$ menor a 0.30$)$, toxicidad mayor al $90 \%$ y alta concentración de materia orgánica (superior a los $1000 \mathrm{mg} / \mathrm{L}$ ) (Ding, Chen y Fan, 2011; Torresano, 2017). En cuanto a los residuos sólidos (RS) de la industria gráfica se pueden clasificar en cuatro grandes grupos: reciclables, no reciclables, peligrosos y de manejo especial (Ministerio de Ambiente y Energía, 2013). Los principales RS son: restos de papel y cartón, trapos de limpieza con disolvente y tinta, planchas utilizadas o defectuosas, contenedores vacíos de disolventes, cartuchos de tinta y tóner, y plástico de empaque dañado (García, Leone y Williams, 2014).

La industria gráfica como cualquier otro flujo industrial debe someterse a un proceso de evaluación de impacto ambiental, esto con el fin de identificar sus principales efectos y de generar medidas preventivas, correctivas y compensatorias hacia el medio ambiente. El proceso de evaluación de impacto ambiental (EIA) es una valoración de los potenciales impactos generados sobre el medio ambiente producto de determinada actividad, obra o proyecto. Es un procedimiento necesario para la valoración de los impactos ambientales de las distintas alternativas de un proyecto determinado, con el fin de definir la mejor opción para su viabilidad (Mora, Molina y Sibaja, 2016).

Diferentes técnicas metodológicas son aplicables al proceso de evaluación ambiental de un proyecto, siendo algunos métodos generales, otros de carácter más 
específico; no obstante, sin importar el método, se pueden extraer técnicas, que con variaciones son muy útiles para este proceso. Aunque algunos métodos o técnicas se diseñaron para proyectos concretos y su adaptación al proyecto de interés es difícil, pueden llegar a ser de gran utilidad (Mora, Molina y Sibaja, 2016).

Evaluaciones de impacto ambiental de procesos litográficos señalan como principales aspectos ambientales los siguientes; (AA): i) emisiones gaseosas proveniente de líquidos volátiles utilizados como diluyentes de tintas (solventes) y limpiadores (García, Leone y Williams, 2014; Dimate y Chirley, 2014); ii) vertido de aguas residuales (Ministerio de Medio Ambiente, 2013; Prica et al 2016) iii) uso de materiales peligrosos y iv) generación de residuos sólidos (Corporacion Financiera internacional, 2007).

En el presente proyecto de investigación se realizó una identificación, valorización y priorización de los principales aspectos ambientales (AAs) e impactos ambientales (IAs) generados en una industria litográfica tipo "offset" en Costa Rica. Se realizaron mediciones ambientales de aguas residuales (AR), compuestos orgánicos volátiles (VOCs), partículas totales en suspensión (PTS), ruido y luminosidad. La identificación de los AAs se realizó en conjunto con los funcionarios de la empresa, con expertos en gestión ambiental y a través de sesiones de trabajo con grupos focales, mediante herramientas como; revisión bibliográfica, lista de chequeo, entrevistas e inspecciones de campo. La valorización y priorización de los AAs identificados se llevó a cabo mediante la metodología denominada Matriz de Importancia de Impacto Ambiental (MIIA).

\section{METODOLOGÍA}

Esta investigación se enfocó en realizar la evaluación del IA en una industria gráfica con el objetivo de estimar si existe un riesgo químico ambiental en el proceso de impresión de la empresa. Esta evaluación se realizó por un periodo de año y un mes (mayo del 2018 y junio del 2019), durante cuatro etapas descritas a continuación:

\section{Identificación de aspectos e impac- tos ambientales}

Mediante la realización de 15 visitas guiadas por funcionarios de la industria gráfica se identificaron las entradas y salidas del proceso productivo y sus principales materias primas e insumos, se estudió el diagrama general del proceso por medio del uso de balances de masa y energía.

Durante las inspecciones de campo se ejecutaron entrevistas, encuestas, listas de chequeo y reuniones con grupos focales de la industria en estudio; también se realizaron con expertos internos (de la empresa) y externos en el tema de evaluación y gestión ambiental, parte de las actividades del trabajo de campo incluyó la realización de talleres y sesiones de trabajo con funcionarios de la planta productiva.

Producto de las actividades mencionadas anteriormente se generó una base de datos con los principales aspectos (AAs) e impactos ambientales (IAs) del proceso productivo de la industria gráfica.

\section{Mediciones de parámetros ambientales}

Con la finalidad de disponer de datos en tiempo real de algunos aspectos ambientales, se realizaron mediciones en campo de la calidad y cantidad de agua residual, 
calidad del aire en ambiente laboral, ruido y cantidad de luminosidad disponible en los diferentes recintos de la industria. El método de muestreo y análisis de cada parámetro se detallan a continuación.

\section{Calidad del aire en ambiente laboral}

Las emisiones medidas en el interior de la planta de producción en la industria gráfica fueron: partículas totales en suspensión (PTS) y compuestos orgánicos volátiles (VOCs).

Las PTS fueron muestreadas utilizando Medidores de Alto Volumen, marca TISCH ENVIRONMENTAL, INC, modelo TE-5000., con un filtro de $(22 \times 28) \mathrm{cm}$ de material de fibra de vidrio, con una medición previa de la masa. El tiempo de muestreo fue de 24 horas, a un flujo promedio de $1 \mathrm{~m}^{3} / \mathrm{min}$. La determinación analítica de la cantidad de partículas se realizó por gravimetría. Este método es descrito en el Método-40 de Referencia de la EPA (U.S. Environmental Protection Agency, 1998). Se realizaron 2 campañas de muestreo en 4 puntos de la industria, donde se ubican las máquinas de impresión (litográfica, numeradora y termocromática) y las máquinas contadoras de papel.
El muestreo de los VOCs se llevó a cabo, mediante cartuchos de adsorción con carbón activado, conectados a bombas con un flujo de $450 \mathrm{~cm}^{3} / \mathrm{min}$ y durante un periodo de 3 horas. La determinación analítica del total de VOCs, se realizó por desorción térmica en un cromatógrafo de gases (GC) marca Perkin Elmer Autosystem XL con detector de ionización de llama (FID). Este método es descrito en el Método-49 de Referencia de la EPA (U. S. Environmental Protection Agency, 1999). Se realizó una campaña de muestreo, en 10 puntos de la industria.

De forma complementaria, para la identificación de los VOCs se tomaron muestras puntuales en 6 puntos de la industria, que utiliza un GC portátil marca Che$\mathrm{mID} \AA$, cuyo programa permite la identificación de sustancias in situ.

\section{Aguas residuales}

Se realizaron 3 campañas de muestreo para la evaluación de la calidad del agua residual mediante el análisis de los parámetros mostrados en la Tabla 1. La muestra se tomó de forma compuesta durante un periodo de tiempo de 1 hora, obteniéndose 5

Tabla 1. Parámetros de análisis en las AR y sus métodos de análisis basados en metodologías del Standard Methods for the Examination of Water and Wastewater. 22nd Ed. 2012

\begin{tabular}{lc}
\hline \multicolumn{1}{c}{ Determinación } & Método de análisis \\
\hline $\mathrm{pH}$ & $4500 \mathrm{HB}$ \\
Sólidos suspendidos totales (SST), mg/L & $2540 \mathrm{D}$ \\
Sólidos sedimentables (SS), mL/L & $2540 \mathrm{~F}$ \\
Grasas y aceites, mg/L & $5520 \mathrm{~B}$ \\
Demanda Química de Oxígeno, DQO mg/L & $5220 \mathrm{D}$ \\
Demanda Bioquímica de Oxígeno, DBO5 $20 \mathrm{mg} / \mathrm{L}$ & $5210 \mathrm{~B}$ \\
Temperatura, ${ }^{\circ} \mathrm{C}$ & $2550 \mathrm{~B}$ \\
Sustancias Activas al Azul de Metileno (SAAM), mg/L & $5540 \mathrm{C}$ \\
Color, \% & $2120 \mathrm{D}$ \\
Metales (arsénico, cadmio, cromo, mercurio y plomo) & $\begin{array}{l}\text { Espectrometría de plasma de acoplamiento } \\
\mathrm{mg} / \mathrm{L}\end{array}$ \\
\hline
\end{tabular}

Nota: Fuente propia de la investigación. 
submuestras de $300 \mathrm{~mL}$. El punto de muestreo correspondió al único punto generador (denominado punto de máquina $\mathrm{CTP}$ ), el cual es la única fuente de AR especiales vertidas al alcantarillado sanitario público.

\section{Ruido}

La medición del ruido se realizó mediante 3 campañas de muestreo en 5 puntos: en los sitios donde se encuentran las máquinas de impresión (litográfica, numeradora y termocromática), el cuarto de conteo de papel y en preimpresión en la máquina CTP. En la medición del ruido se empleó un dosímetro sónico marca EXTECH ${ }^{\circledR}$ modelo HD600, con filtro tipo A y se realizó las mediciones con base al valor laboral permitido $85 \mathrm{~dB}$ en base 10 .

\section{Luminosidad}

La intensidad luminosa se midió en 5 puntos de la industria para lo cual se empleó un luxómetro MTP $\AA$ modelo 1210. Se realizaron 3 campañas de muestreo. Los puntos de muestreos son; 1) control de calidad, 2) bóveda, 3) oficinas, 4) preimpresión (CTP) y 5) impresión de numeración.

\section{Valorización de aspectos e impactos ambientales}

La valoración del IA de cada AAs se realizó con la matriz de Importancia de Impacto Ambiental (MIIA) descrita en el decreto costarricense 32966 (Ministerio de Ambiente Y Energía y Ministerio de Salud, 2006). La escogencia de los criterios a incluir en la MIIA se realizó mediante la información recopilada en el trabajo de campo, sesiones de trabajo con funcionarios, la jefatura de la industria gráfica, la encargada de ambiente y 4 expertos en el área de gestión ambiental. La pluralidad de criterios permito eliminar la subjetividad en la inclusión de los datos en la matriz de evaluación ambiental. Los resultados obtenidos producto de la medición en campo de los parámetros ambientales se utilizaron como insumos para la MIIA. En la Tabla 2 se incluyen los factores de evaluación (signo, extensión, intensidad, momento, persistencia, reversibilidad, recuperabilidad, acumulación, sinergia, efecto y periodicidad) que utiliza la matriz MIAA para la evaluación del impacto ambiental. Se incluye además en la tabla el criterio de campo utilizado en la presente investigación para definir el valor del factor incluido en la matriz MIIA.

Priorización de aspectos e impactos ambientales

El valor de importancia del impacto ambiental de cada aspecto ambiental se determinó mediante la siguiente ecuación

$$
\begin{gathered}
I= \pm(3 I N+2 E X+M O+P E+P V+ \\
S I+A C+E F+P R+M C)
\end{gathered}
$$

La priorización de cada AA e IA se realizó mediante las escalas de valoración de impacto e importancia del impacto incluidas en la Tabla 3. De esta forma un impacto ambiental puede ser clasificado en; irrelevante negativo, crítico negativo, severo positivo, irrelevante positivo, entre otros.

\section{ANÁLISIS Y RESULTADOS}

\section{Identificación de aspectos e impac- tos ambientales}

Se identificaron 16 AA y 5 IA, los AA se clasifican en 5 categorías generales en función al medio o persona que pueden afectar. Las principales fuentes 

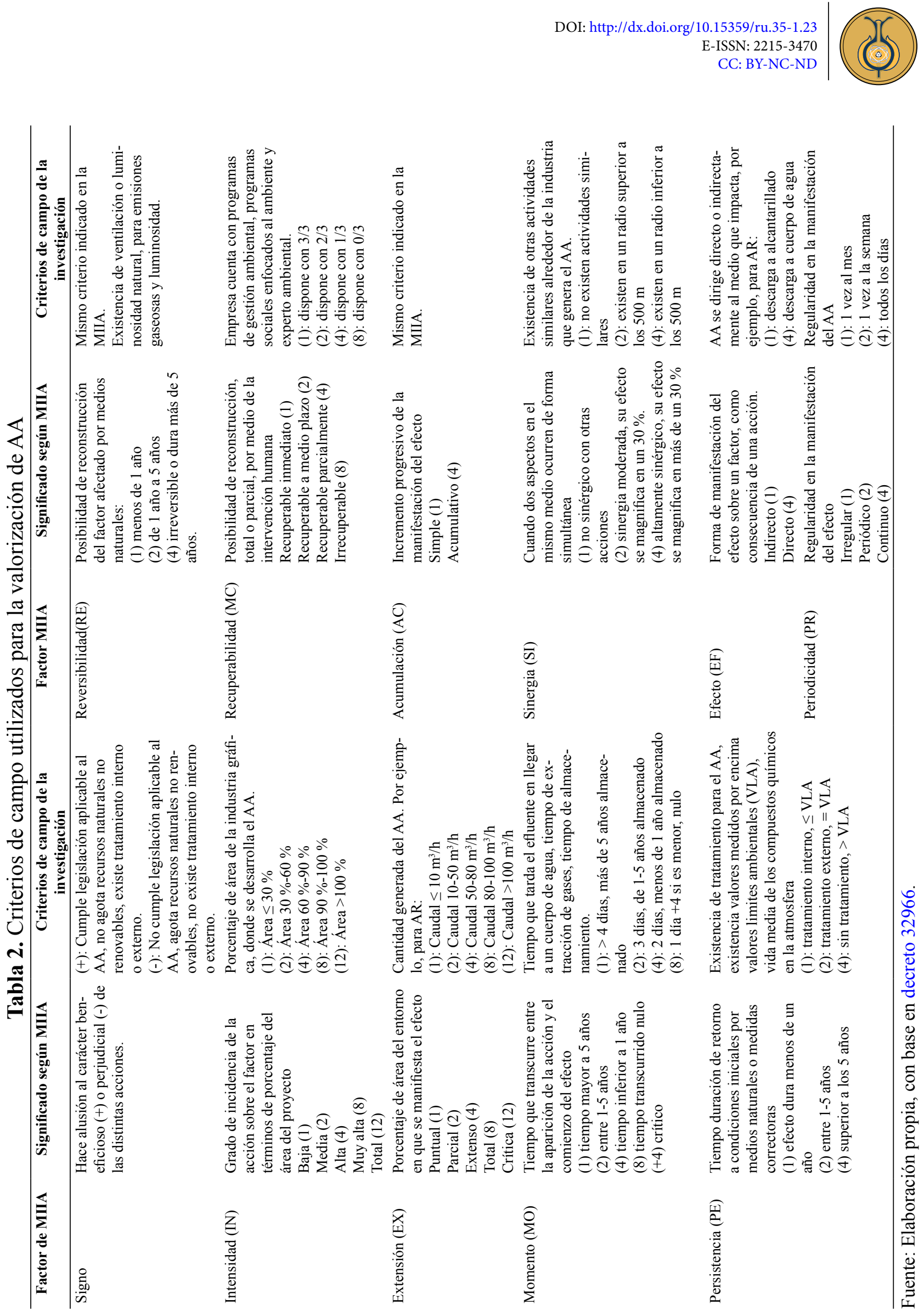
Tabla 3. Interpretación de los valores de importancia del impacto, de acuerdo con el rango de la puntuación obtenida para cada aspecto o impacto ambiental

\begin{tabular}{|c|c|c|c|}
\hline Importancia & Rango & Color & Priorización \\
\hline \multicolumn{4}{|c|}{ Valores de importancia signo negativo } \\
\hline Irrelevante negativo & $<-25$ & & Bajo \\
\hline Moderado negativo & $-25 a-50$ & & Medio \\
\hline Severo negativo & $-50 a-75$ & & Alto \\
\hline Crítico negativo & $>-75$ & & Muy alto \\
\hline \multicolumn{4}{|c|}{ Valores de importancia signo positivo } \\
\hline Critico positivo & $<+25$ & & Muy alto \\
\hline Severo positivo & $+25 \mathrm{a}+50$ & & Alto \\
\hline Moderado positivo & $+50 \mathrm{a}+75$ & & Medio \\
\hline Irrelevante positivo & $>+75$ & & Bajo \\
\hline
\end{tabular}

Nota: Fuente decreto 32966.

generadoras de aspectos ambientales son las aguas residuales, los residuos sólidos, las emisiones a la atmósfera, el ruido, la luminosidad, el uso de sustancias químicas y el consumo de agua, papel y madera. En la Tabla 4, se incluye el aspecto ambiental y su fuente de generación, así como el impacto ambiental generado.

En el proceso se distinguen 2 tipos de AR: ordinarias y especiales (que contienen productos químicos). Sin embargo, para efectos de este trabajo, las aguas residuales especiales se dividieron en 2 categorías: AR del proceso de generación de la plancha y AR con solventes y tintas. La diferencia está en la forma de disposición final de las mismas, ya que las AR del proceso de planchado se desfogan a través del alcantarillado sanitario y las AR con solventes y tintas, son separadas en la fuente y enviadas a incineración a través de un gestor externo.

\section{Resultados de la medición de pará- metros ambientales}

Durante el periodo comprendido entre mayo del 2018 y junio del 2019, se analizaron parámetros ambientales como: AR, ruido, luminosidad y calidad del aire en una industria gráfica en Costa Rica; con el fin de conocer su situación ambiental actual y tomarla en cuenta para la valorización de AAs e IAs.

\section{Aguas residuales}

Los resultados obtenidos para el único punto de muestreo de aguas residuales se incluyen en la Tabla 5. Según los datos anteriores, todas las mediciones realizadas se encuentran dentro de los límites establecidas por el decreto 33601-S. Sin embargo, es importante indicar que la medición se realizó en las AR provenientes de la máquina CTP, debido al desconocimiento por parte de funcionarios del desfogue final de AR hacia el sistema de alcantarillado público.

Las concentraciones de DQO fueron superiores a las de DBO encontradas, con un índice de biodegradabilidad promedio de $0.39\left(\mathrm{DBO}_{5} / \mathrm{DQO}\right)$, lo cual es coincidente con lo indicado en la bibliografía (Prica et al, 2016; Ding, Chen y Fan, 2011; Torresano, 2017).

\section{Calidad del aire en ambiente laboral}

La calidad del aire en el ambiente laboral constituye un aspecto importante a tomar en cuenta en la evaluación del IA de 
Tabla 4. Inventariado de los aspectos e impactos ambientales identificados en la industria gráfica

\begin{tabular}{|c|c|c|c|}
\hline Categoría & Aspecto ambiental & Fuente de generación & Impacto ambiental \\
\hline \multirow{3}{*}{$\begin{array}{l}\text { Aguas } \\
\text { Residuales y } \\
\text { Vertidos }\end{array}$} & $\begin{array}{l}\text { Generación de aguas residuales } \\
\text { especiales }\end{array}$ & $\begin{array}{l}\text { Provenientes del proceso de planchado que } \\
\text { contienen sustancias peligrosas }\end{array}$ & \multirow{3}{*}{$\begin{array}{l}\text { Contaminación de } \\
\text { aguas superficiales y } \\
\text { subterráneas }\end{array}$} \\
\hline & $\begin{array}{l}\text { Generación de aguas residuales } \\
\text { con solventes y tintas }\end{array}$ & $\begin{array}{l}\text { Almacenamiento de AR con sustancias } \\
\text { peligrosas }\end{array}$ & \\
\hline & $\begin{array}{l}\text { Generación de aguas residuales } \\
\text { ordinarias }\end{array}$ & $\begin{array}{l}\text { Aguas negras y grises provenientes de } \\
\text { servicios sanitarios y pilas }\end{array}$ & \\
\hline \multirow{4}{*}{$\begin{array}{l}\text { Residuos } \\
\text { sólidos }\end{array}$} & Generación de RS reciclables & Disposición de papel, cartón y planchas & \multirow{4}{*}{$\begin{array}{l}\text { Contaminación del } \\
\text { suelo }\end{array}$} \\
\hline & Generación de RS no reciclables & $\begin{array}{l}\text { Disposición de flejes, plástico de empaque, } \\
\text { afiches y membrana protectora }\end{array}$ & \\
\hline & Generación de RS peligrosos & $\begin{array}{l}\text { Disposición de envases plásticos y metálicos, } \\
\text { trapos y cartón con productos químicos y tintas }\end{array}$ & \\
\hline & Generación de RS especiales & $\begin{array}{l}\text { Disposición de luminarias, baterías y } \\
\text { electromecánicos }\end{array}$ & \\
\hline \multirow{2}{*}{$\begin{array}{l}\text { Emisiones a la } \\
\text { atmósfera }\end{array}$} & Emisiones gaseosas de VOCs & Uso de disolventes con químicos volátiles & \multirow{2}{*}{$\begin{array}{l}\text { Contaminación de la } \\
\text { atmósfera }\end{array}$} \\
\hline & Emisión de gases de combustión & $\begin{array}{l}\text { Uso de montacargas para movimiento de } \\
\text { materia prima. }\end{array}$ & \\
\hline \multirow[t]{3}{*}{$\begin{array}{l}\text { Ambiente } \\
\text { laboral }\end{array}$} & Generación de ruido & $\begin{array}{l}\text { Maquinas: Speed Máster, numeradora, } \\
\text { Termocromática y CTP }\end{array}$ & \multirow[t]{3}{*}{$\begin{array}{l}\text { Afectaciones a la } \\
\text { salud }\end{array}$} \\
\hline & Luminosidad & $\begin{array}{l}\text { Control de calidad visual y generación del } \\
\text { arte de los billetes }\end{array}$ & \\
\hline & $\begin{array}{l}\text { Uso de sustancias químicas } \\
\text { peligrosas }\end{array}$ & Disolventes, gomas, revelador y tintas & \\
\hline \multirow[t]{4}{*}{$\begin{array}{l}\text { Recursos no } \\
\text { renovables }\end{array}$} & Consumo energético & $\begin{array}{l}\text { Utilización de maquinaria con altas } \\
\text { demandas de consumo energético }\end{array}$ & \multirow[t]{4}{*}{$\begin{array}{l}\text { Agotamiento de } \\
\text { recursos naturales }\end{array}$} \\
\hline & Consumo de papel & Papel de impresión y billete & \\
\hline & Consumo de agua & Agua para producción y uso general & \\
\hline & Consumo de madera & Tarimas y tapas de madera & \\
\hline
\end{tabular}

Nota: Fuente propia de la investigación.

Tabla 5. Valores medidos de los parámetros de AR en la industria gráfica. Promedio y desviación

\begin{tabular}{|c|c|c|c|c|c|c|}
\hline Parámetro & Muestreo 1 & Muestreo 2 & Muestreo 3 & Promedio & Desviación & $\begin{array}{c}\text { Valor } \\
\text { Normativa* }\end{array}$ \\
\hline $\mathrm{pH}, \pm 0.04$ & 6.84 & 7.66 & 7.10 & 7.20 & 0.42 & 6 a 9 \\
\hline $\begin{array}{l}\text { Solidos Suspendidos Totales, } \pm \\
6.0 \mathrm{mg} / \mathrm{L}\end{array}$ & 24.0 & 8.0 & 13.5 & 15.2 & 8.1 & 300 \\
\hline Solidos Sedimentables, $\mathrm{mL} / \mathrm{L}$ & $<0.2$ & $<0.13$ & $<0.53$ & NA & NA & 1.5 \\
\hline Grasas y Aceites, mg/L & $<3$ & $<5$ & $<5$ & NA & NA & 50 \\
\hline $\mathrm{DQO}, \pm 6.0 \mathrm{mg} / \mathrm{L}$ & 136.0 & 175.0 & 98.0 & 136.3 & 38.5 & 750 \\
\hline $\mathrm{DBO}_{520}, \pm 4.0 \mathrm{mg} / \mathrm{L}$ & 38.0 & 90.0 & 33.2 & 53.7 & 31.5 & 300 \\
\hline Temperatura, $\pm 0.3^{\circ} \mathrm{C}$ & 26.2 & 23.0 & 27.3 & 25.5 & 2.3 & $\begin{array}{l}15^{\circ} \mathrm{C} \leq \mathrm{T} \leq \\
40^{\circ} \mathrm{C}\end{array}$ \\
\hline Caudal Volumétrico, $\pm 0.02 \mathrm{~m}^{3} / \mathrm{h}$ & 0.44 & 0.14 & 0.17 & 0.25 & 0.17 & --- \\
\hline $\begin{array}{l}\text { Sustancias Activas al Azul de } \\
\text { Metileno, } \pm 0.10 \mathrm{mg} / \mathrm{L}\end{array}$ & 4.31 & 4.90 & 1.27 & 3.49 & 1.95 & 7.5 \\
\hline Color, $\%$ & 11.0 & $<1$ & $<1$ & NA & NA & 15 \\
\hline
\end{tabular}

* Decreto N. ${ }^{\circ}$ 33601-MINAE-S, Reglamento de Vertido y Reuso de Aguas Residuales, La Gaceta 55, Alcance 8 del lunes 19 de marzo de 2007

Nota: Fuente propia de la investigación. 
cualquier proceso productivo (Occupational Safety and Health Branch Labour Department, 2004). Las emisiones generadas en la industria gráfica corresponden a PTS, VOCs y gases de combustión. En la Tabla 6 se detallan los resultados de los muestreos de PTS y VOCs realizados en 10 puntos de muestreo. Los valores de los parámetros medidos se reportan según condiciones estándar de temperatura y presión.

El Institutito Nacional de Seguridad, Salud y Bienestar en el Trabajo de España define el valor límite ambiental de exposición diaria (VLA-ED) como la concentración media del agente químico en la zona de respiración del trabajador medida con respecto al tiempo, para una jornada laboral estándar de 8 horas diarias. El VLA-ED definido para la concentración de PTS y VOCS es de $3000 \mu \mathrm{g} / \mathrm{Sm}^{3}$ y $160 \mu \mathrm{g} / \mathrm{Sm}^{3}$, respectivamente (INSSBT, 2019). Según los resultados incluidos en la Tabla 6, los valores de VOCs para todos los puntos de muestreo se ubican entre $292 \mu \mathrm{g} / \mathrm{m}^{3}$ y $4047 \mu \mathrm{g} / \mathrm{m}^{3}$ ), por lo tanto existe un riesgo hacia la salud de los trabajadores como producto de la exposición.
El inventario in situ de los VOCs analizados mediante el cromatógrafo de gases portátil, determinó, en algunos puntos de muestreos, compuestos químicos como tolueno y bromoformo con resultados superiores al VLA-ED. Para el tolueno se reportan valores superiores al permitido $(50.0 \mathrm{ppm})$ en los puntos de muestreo denominados: a) máquina CTP (123.0), en litografía (73.3), b) oficina del taller (114.0) y c) conteo de papel (100.0). Los valores reportados se deben principalmente al uso de thinner utilizado en la limpieza de las máquinas de impresión, así como el uso de otros solventes con contenido de compuestos aromáticos.

Otro solvente orgánico detectado durante el muestreo con el equipo portátil es el bromoformo, su concentración ambiental supera el valor permitido $(0.5 \mathrm{ppm})$ en el punto de muestreo denominado oficina del taller $(3.25 \mathrm{ppm})$. En el inventario de los productos químicos utilizados en el proceso productivo no existe producto químico que contenga bromoformo en su composición química; no obstante, no se descarta que la fuente sea algún producto de limpieza y

Tabla 6. Valores medidos en el muestreo interno de VOCs y PTS

\begin{tabular}{|c|c|c|c|c|c|}
\hline \multirow{2}{*}{$\begin{array}{c}\text { Medición } \\
\text { Punto de muestreo }\end{array}$} & \multicolumn{3}{|c|}{ PTS $\mu \mathrm{g} / \mathrm{Sm}^{3}$} & \multicolumn{2}{|c|}{ VOCs-CH4 $\mu \mathrm{g} / \mathrm{Sm}^{3}$} \\
\hline & Muestreo 1 & Muestreo 2 & VLA-ED & Muestreo 1 & VLA-ED \\
\hline Oficinas & *NA & *NA & 3000 & $318 \pm 46$ & 160 \\
\hline Comedor & *NA & *NA & & $292 \pm 43$ & \\
\hline Bóveda & *NA & *NA & & $681 \pm 101$ & \\
\hline Control de calidad & *NA & $* \mathrm{NA}$ & & $388 \pm 57$ & \\
\hline Termocromática & $35 \pm 3$ & $29 \pm 2$ & & $801 \pm 119$ & \\
\hline Litografía & $36 \pm 3$ & $40 \pm 4$ & & $4047 \pm 607$ & \\
\hline CTP & *NA & *NA & & $1729 \pm 259$ & \\
\hline Numeradora & $36 \pm 3$ & $32 \pm 3$ & & $983 \pm 147$ & \\
\hline Ventas & $* \mathrm{NA}$ & $* \mathrm{NA}$ & & $2884 \pm 440$ & \\
\hline Conteo de papel & $20 \pm 2$ & $24 \pm 2$ & & $2669 \pm 400$ & \\
\hline Oficina del taller producción & *NA & *NA & & $3516 \pm 527$ & \\
\hline
\end{tabular}

*NA: no aplica, no se realizó el muestreo en este punto.

Nota: Fuente propia de la investigación. 
desinfección. El bromoformo se caracteriza por contener una alta toxicidad humana registrada (INSSBT, 2019).

\section{Ruido}

Los valores obtenidos de la medición del ruido (Tabla 7 ), indican que al menos dos puntos sobrepasan el VL permitido (85) según decreto N. ${ }^{\circ} 10541-T S S:$ Reglamento para el control de ruidos y vibraciones.

Según la OMS, la industria de las artes gráficas se ubica en el puesto número 8 dentro de los 20 tipos de actividades con mayor generación de ruido, entre los efectos del ruido están: la pérdida de la capacidad auditiva, acúfenos (sensación de zumbido en los oídos), la interferencia en la comunicación hablada y en la percepción de las señales de alarma, las alteraciones del rendimiento laboral, las molestias y los efectos extra auditivos (Enciclopedia de salud y seguridad en el Trabajo, 2019).
Las mediciones se realizaron en los lugares donde hay presencia de máquinas que generan ruido. Según la Tabla 7, cuatro de los sitios muestreados presentan valores por encima de $80 \mathrm{~dB}$. Esto se debe a que las máquinas numeradora, termocromática, litográfica y las contadoras, realizan procesos rápidos y continuos, que generan ruido y vibraciones. El punto de muestreo de la máquina CTP muestra valores de ruido menores al permitido, esto se debe a que el proceso de generación de la plancha es más pasivo: se graban las imágenes en esta, la misma se pasa por un revelador, engomado y lavado.

\section{Luminosidad}

La cantidad de luz disponible para llevar a cabo una función laboral es indispensable para su buen desempeño (Norma INTE 31-08-06-2000). En la Tabla 8, se muestran los valores de luminosidad obtenidos en las diferentes zonas de la industria gráfica.

Tabla 7. Valores medidos de ruido por punto de muestro en el taller de la industria gráfica

\begin{tabular}{lcccccc}
\hline & \multicolumn{9}{c}{ Ruido \pm 0.1 dB10 } & & & \\
Punto de muestreo & M 1 & M 2 & M 3 & Promedio & Desviación & Valor normativo* \\
\hline Máquina CTP & 61.9 & 70.4 & 56.1 & 62.8 & 7.2 & 85 \\
Litografía & 87.2 & 89.3 & 86.9 & 87.8 & 1.3 & \\
Termocromática & 81.4 & 81.9 & 84.6 & 82.6 & 1.7 & \\
Contadoras de papel & 79.8 & 83.3 & 82.0 & 81.7 & 1.8 & \\
Numeradora & - & 85.5 & 85.1 & 85.3 & 0.3 &
\end{tabular}

*Valor obtenido del Decreto N. ${ }^{\circ}$ 10541-TSS: Reglamento para el control del ruido.

Nota: Fuente propia de la investigación.

Tabla 8. Valores medidos de luminosidad en la industria gráfica

\begin{tabular}{lcccccc}
\hline \multicolumn{1}{c}{ Lugar } & \multicolumn{2}{c}{ Luminosidad $\pm \mathbf{0 . 0 1}$ lux } & & & \\
& & M2 & M3 & Promedio & Desviación & $\begin{array}{c}\text { Valor } \\
\text { recomendado* }\end{array}$ \\
\hline Control de cal- & 426.00 & 425.00 & 416.67 & 422.56 & 5.12 & 1000 \\
idad & & & & & & \\
Bóveda & 501.00 & 492.00 & 482.33 & 491.78 & 9.34 & 1000 \\
Oficinas & 239.46 & 165.90 & 165.70 & 190.35 & 42.53 & 500 \\
Máquina CTP & 80.74 & 87.00 & 79.43 & 82.39 & 4.05 & 500 \\
Numeradora & 390.24 & 323.10 & 378.3 & 363.88 & 35.82 & 1000 \\
\hline
\end{tabular}

*Valor obtenido de Norma INTE 31-08-06-2000.

Nota: Fuente propia de la investigación. 
La mayoría de las áreas de trabajo del taller de impresión son cerrados y con baja luminosidad natural, por lo tanto, la variación entre los datos de luminosidad obtenidos se debe a la mayor o menor cantidad disponible de luz artificial. Según la Norma INTE 31-0806-2000 los valores medidos de luminosidad son bastante bajos. Esta hace referencia a los niveles y condiciones de iluminación que deben tener los centros de trabajo, la norma indica que el valor mínimo de luminosidad en la industria litográfica es de 700 lux.

\section{Valorización y priorización de los aspectos y los impactos ambientales}

Un ejemplo de la valoración de los AAs según lo contenido en la Tabla 2 y de la evaluación del aspecto ambiental: Generación de aguas residuales especiales en particular, se muestra en la Tabla 9.

El valor de importancia del impacto ambiental de cada aspecto ambiental evaluado según la Matriz MIIA utilizando los criterios de campo incluidos la Tabla 2, se detallan en el gráfico siguiente y en la Tabla 10.
Los valores de los AAs indican que se tienen; cuatro AA con un IA severo positivo, un AA con un IA moderado positivo, un AA con un IA irrelevante positivo, dos AA con IA moderado negativo, cinco AA con IA severo negativo y tres AA con IA critico negativo. Del total (10) de AAs negativos, dos AAs fueron priorizados con un valor de impacto de medio, un total de cinco AAs de alto y tres son de prioridad de acción muy alta, es decir la industria debe tomar medidas inmediatas para disminuir su IA.

En la determinación del valor del impacto final de cada AA (ecuación 1) los factores de extensión e intensidad son los que más aportan, numéricamente, al valor final del impacto. Por lo tanto, AAs con valores altos de extensión e intensidad tendrán los mayores valores positivos o negativos del respectivo IA.

Según el Gráfico 1 y la Tabla 10, el AA con mayor impacto positivo al medio ambiente es la generación de RS reciclables, el cual tiene un valor de impacto de +86 (contaminación del suelo), este impacto

Tabla 9. Ejemplo para la evaluación del aspecto aguas residuales especiales en la industria gráfica

\begin{tabular}{|c|c|c|}
\hline Factor & Valor & Justificación \\
\hline Signo & + & Cumple decreto 33601, según la tabla 5 \\
\hline Intensidad & 1 & $\begin{array}{l}\text { AR provenientes de la maquina CTP del subproceso preimpresión: } \leq 30 \% \text { del } \\
\text { área de la industria gráfica }\end{array}$ \\
\hline Extensión & 1 & Se observa en la tabla 5 , que el caudal de vertido no supera los $10 \mathrm{~m}^{3} / \mathrm{h}$. \\
\hline Momento & 8 & $\begin{array}{l}\text { El efluente dura } 1 \text { día para ingresar a el cuerpo de agua, según datos del Instituto } \\
\text { de Acueductos y Alcantarillados (AyA) de Costa Rica }\end{array}$ \\
\hline Persistencia & 2 & Existe tratamiento externo en una planta de tratamiento del AyA \\
\hline Reversibilidad & 1 & $\begin{array}{l}\text { Caudal bajo de vertido y presencia de tratamiento, se considera que su tiempo de } \\
\text { remoción no será mayor a } 1 \text { año }\end{array}$ \\
\hline Recuperabilidad & 4 & $\begin{array}{l}\text { Industria no se cuenta con programas de acción social o con un profesional en } \\
\text { temas ambientales que guíe a la empresa en acciones en pro del ambiente }\end{array}$ \\
\hline Acumulación & 4 & AA es acumulativo, incremento de la manifestación del efecto \\
\hline Sinergia & 4 & Se consideró que a $300 \mathrm{~m}$ a la redonda de la industria hay actividades similares \\
\hline Efecto & 1 & Indirecto por existencia de tratamiento previo antes de vertido a cuerpo de agua \\
\hline Periodicidad & 4 & Proceso productivo continuo \\
\hline
\end{tabular}

Nota: Fuente propia de la investigación. 


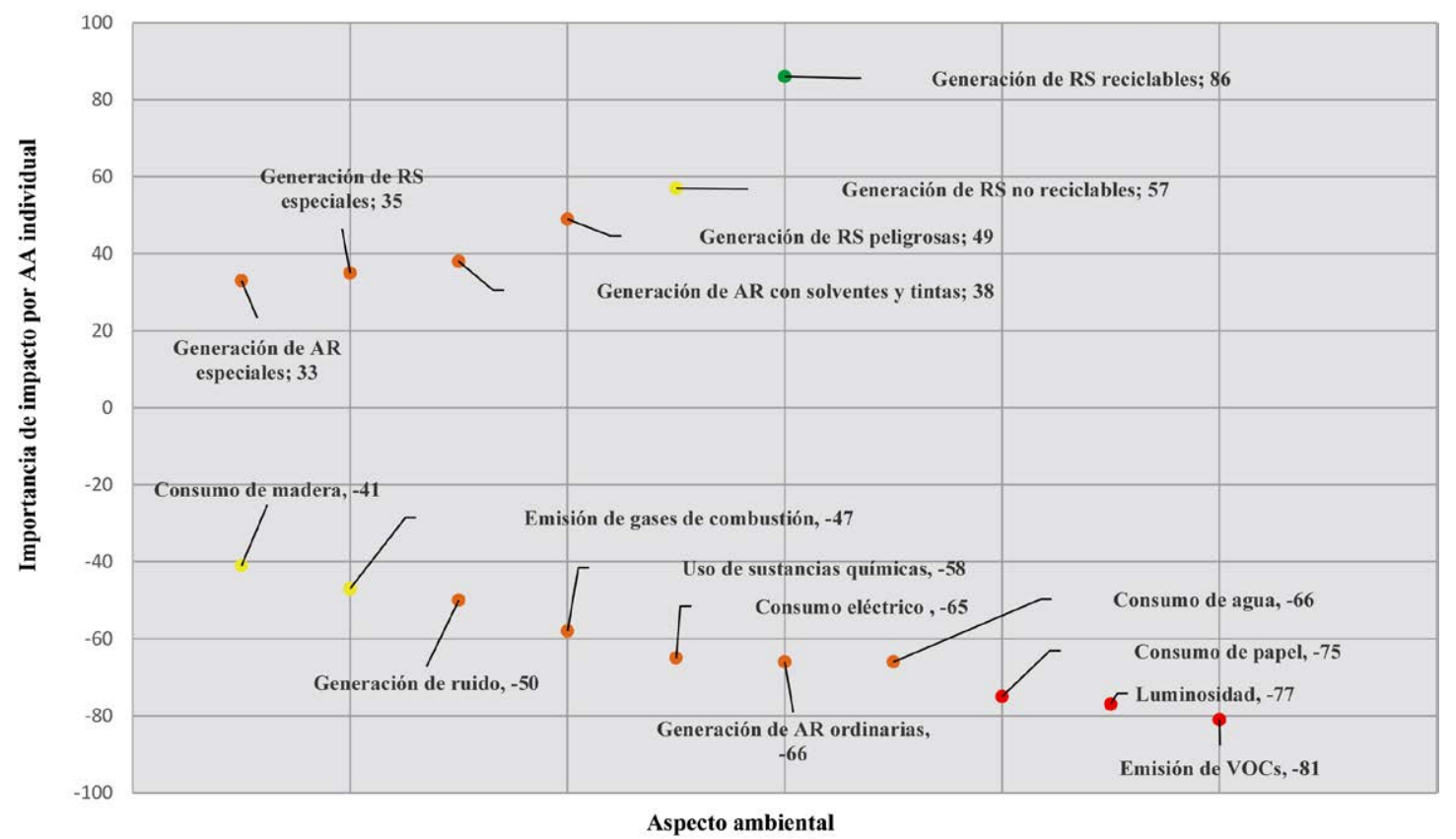

Gráfica 1. Distribución de valores de importancia de impacto por aspecto ambiental individual *Colores asignados según categorías en la Tabla 3.

Nota: Fuente propia de la investigación.

Tabla 10. Resumen de importancia y priorización de impactos ambientales (Valor individual)

\begin{tabular}{|c|c|c|c|c|c|c|}
\hline \multirow[t]{2}{*}{ Aspecto ambiental } & \multicolumn{5}{|c|}{ Importancia del impacto ambiental positivos (MIIA) } & \multirow{2}{*}{$\begin{array}{l}\text { Priorización del } \\
\text { impacto }\end{array}$} \\
\hline & Impacto Ambiental & Valor & Importancia & Rango & Color & \\
\hline $\begin{array}{l}\text { Generación de aguas residuales } \\
\text { especiales }\end{array}$ & \multirow{2}{*}{$\begin{array}{l}\text { Contaminación de aguas } \\
\text { superficiales y subter- } \\
\text { ráneas }\end{array}$} & +33 & \multirow[t]{4}{*}{ Severos positivos } & \multirow[t]{4}{*}{$+25 \mathrm{a}+50$} & & \multirow[t]{4}{*}{ Alta } \\
\hline $\begin{array}{l}\text { Generación de aguas residuales } \\
\text { con solventes y tintas }\end{array}$ & & +38 & & & & \\
\hline Generación de RS peligrosos & \multirow[t]{4}{*}{ Contaminación del suelo } & +49 & & & & \\
\hline Generación de RS especiales & & +35 & & & & \\
\hline Generación de RS no reciclables & & +57 & Moderados positivos & $+50 \mathrm{a}+75$ & & Media \\
\hline Generación de RS reciclables & & +86 & Irrelevantes positivos & $>+75$ & & Baja \\
\hline \multicolumn{7}{|c|}{ Importancia del impacto ambiental negativo (MIIA) } \\
\hline Emisión de gases de combustión & $\begin{array}{l}\text { Contaminación de la } \\
\text { atmósfera }\end{array}$ & -47 & Moderados negativos & $\begin{array}{l}-25 a \\
-50\end{array}$ & & \multirow[t]{2}{*}{ Media } \\
\hline Consumo de madera & $\begin{array}{l}\text { Agotamiento de recursos } \\
\text { no renovables }\end{array}$ & -41 & & & & \\
\hline $\begin{array}{l}\text { Generación de aguas residuales } \\
\text { ordinarias }\end{array}$ & $\begin{array}{l}\text { Contaminación de aguas } \\
\text { superficiales y subter- } \\
\text { ráneas }\end{array}$ & -66 & Severos negativos & $\begin{array}{l}-50 \mathrm{a} \\
-75\end{array}$ & & \multirow[t]{5}{*}{ Alta } \\
\hline Generación de ruido & \multirow[t]{2}{*}{ Afectación a la salud } & -50 & & & & \\
\hline Uso de sustancias químicas & & -58 & & & & \\
\hline Consumo eléctrico & \multirow{2}{*}{$\begin{array}{l}\text { Agotamiento de recursos } \\
\text { naturales }\end{array}$} & -65 & & & & \\
\hline Consumo de agua & & -66 & & & & \\
\hline Emisión de VOCs & $\begin{array}{l}\text { Contaminación de la } \\
\text { atmósfera }\end{array}$ & -81 & Críticos negativos & $>-75$ & & \multirow[t]{3}{*}{ Muy alta } \\
\hline Luminosidad & Afectación a la salud & -77 & & & & \\
\hline Consumo de papel & $\begin{array}{l}\text { Agotamiento de recursos } \\
\text { naturales }\end{array}$ & -75 & & & & \\
\hline
\end{tabular}

Nota: Fuente propia de la investigación. 
es considerado "irrelevante positivo" y de prioridad baja. El impacto positivo de este AA se debe a que en la en la industria los RS son inventariados y tratados mediante un gestor externo.

Entre otros AA con valores de impacto beneficioso al medio ambiente están; generación de RS no reciclables $(+57)$, generación de $\mathrm{RS}$ peligrosos $(+49)$ y generación de AR con disolventes y tintas (+38). Estos aspectos generan los siguientes impactos respectivamente: contaminación del suelo y contaminación de aguas superficiales y subterráneas. El valor positivo del impacto de cada uno de estos AA obedece a que estos residuos son generados solo en el taller de impresión tras obtener un valor de 2 en el factor intensidad. Además, la cantidad AR y RS peligrosos producidos al mes son de $557 \mathrm{~L} / \mathrm{mes}$ y $100 \mathrm{~kg} / \mathrm{mes}$, respectivamente, lo cual es un valor relativamente bajo si se compara con otras actividades industriales (García, Leone y Williams, 2014). Según el criterio de extensión de la matriz MIA los valores asignados son de 4 y 8 , en forma correspondiente. Por otro lado, estos AAs son de carácter positivos debido a que la empresa gestiona estos residuos mediante tratamientos externos autorizados. Lo cual se considera, una práctica considerada adecuada (García, Leone y Williams, 2014). En el caso particular de las aguas residuales con solventes y tintas, su valor obedece particularmente al control interno de separación en la fuente: una vez que se genera el AR con tintas y/o disolventes se añaden a estañones identificados con anticipación.

Al contrario, el AA con mayor impacto negativo al ambiente es la emisión de VOCs, el cual tiene un valor de impacto de -81 (contaminación de la atmósfera), el cual es considerado "critico negativo" y de prioridad muy alta. El impacto negativo de este
AA responde a que, en la industria gráfica se utilizan productos químicos con contenido de líquidos volátiles, que aportan VOCs a la atmósfera interna del lugar. Además, actualmente la empresa no cuenta con equipos de ventilación y extracción general o localizada para los gases producidos, lo que sugiere escaza gestión y control del AA.

Entre otros aspectos ambientales con valores de impacto negativo al medio ambiente están; luminosidad ( -77$)$, consumo de papel (-75), consumo de agua (-66), generación de AR ordinarias y consumo eléctrico (-65). Estos aspectos ambientales presentan impactos como; afectación a la salud, consumo de recursos no renovables y contaminación de aguas superficiales y subterráneas, respectivamente. Los valores negativos de los impactos ambientales se deben, principalmente, al incumplimiento de la Norma INTE-31-08-06-00 para el AA luminosidad, al consumo de recursos no renovables para los AAs consumo de agua, electricidad y papel; y al inadecuado control y seguimiento en los análisis de las AR ordinarias. Se desconoce por parte de la empresa el punto exacto del desfogue final de las aguas residuales hacia el sistema del alcantarillado público.

Es importante resaltar otros aspectos ambientales de valor medio de impacto negativo como lo son, la generación de ruido y uso de sustancias químicas con valores de $-50 \mathrm{y}$ -58 respectivamente, de impacto en la salud. Estos dos aspectos ambientales son clasificados de impacto "severo negativo" y de prioridad alta. El valor del impacto generado por estos aspectos obedece, en específico, al ruido producido por las máquinas de impresión (litográfica, termocromática y numeradora) y contadoras de papel billete; y al uso de sustancias químicas con características toxicológicas perjudiciales para la salud. 
En el Gráfico 2 incluye información sobre el valor promedio del impacto ambiental procedente de los diferentes aspectos ambientales que lo conforman. Los principales impactos ambientales (valor promedio) de carácter negativo son contaminación de la atmósfera, afectación a la salud y agotamiento de recursos no renovables, con valores promedios de $-64,-62$ y -62 , proporcionalmente.

De acuerdo a el Gráfico 2, existe una diferencia numérica importante entre el valor de impacto individual por AA y el IA promedio, por ejemplo en la categoría aguas residuales los valores de impacto individual son $+33,+38$ y -66 , para los AAs: AR especiales, AR con solventes y tintas y AR ordinarias, respectivamente; y el valor final promedio del IA es 1.7. No obstante, existen impactos como: contaminación a la atmósfera, afectación a la salud y agotamiento de recursos no renovables que presentan altos valores de impacto, tanto en su valoración individual como en su valoración de IA promedio.

El impacto contaminación de la atmósfera es el IA negativo más significativo (-64), esto se debe al constante uso de productos que contienen VOCs, y a la falta de equipos de tratamiento general o localizados, para los gases emitidos.

\section{CONCLUSIONES}

El muestreo de los parámetros ambientales (AR, ruido, luminosidad y VOCs) permitieron conocer el estado actual y

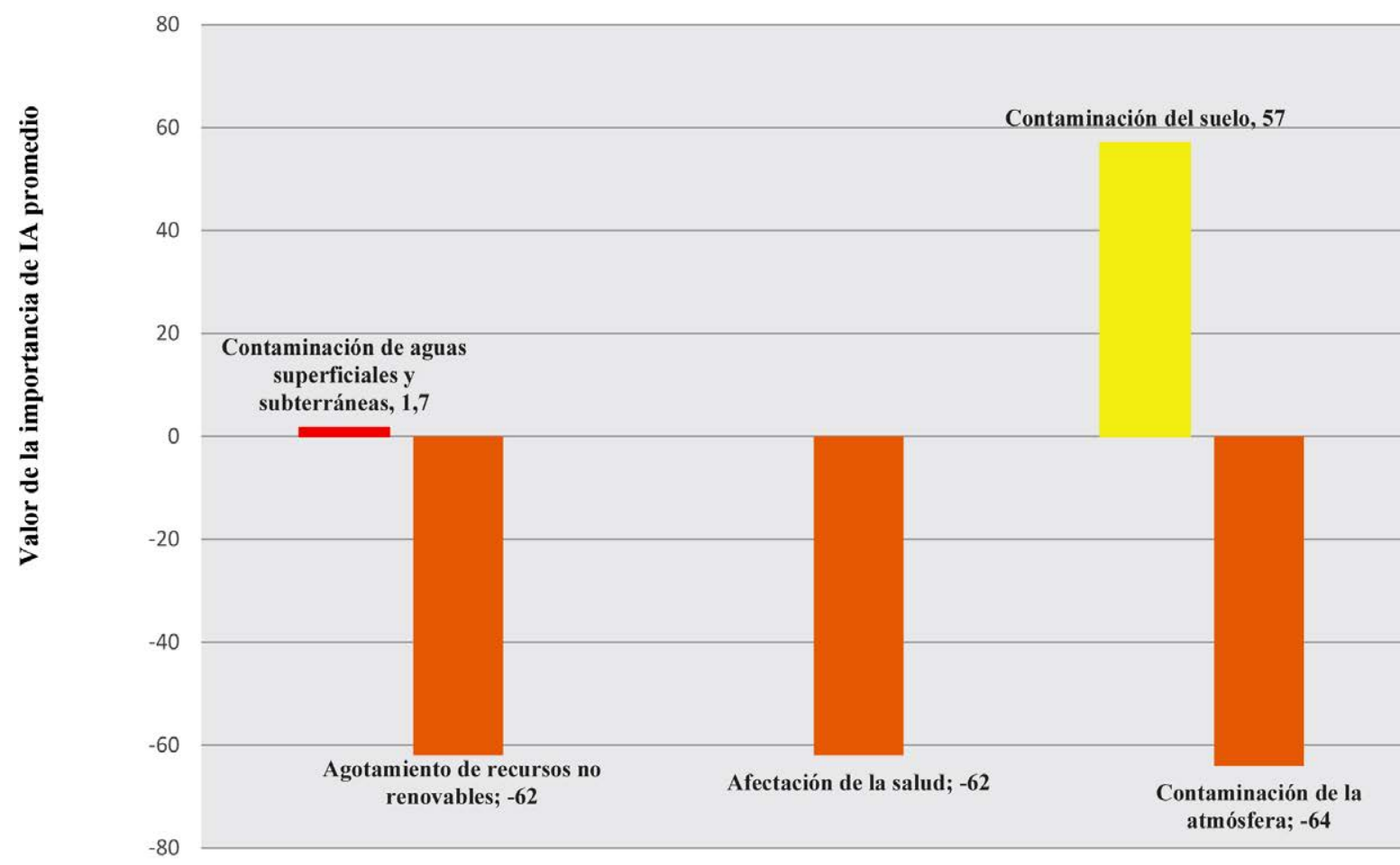

Impacto ambiental

Gráfica 2. Valores promedio de importancia de impacto ambiental

*Colores asignados según categorías de la Tabla 3.

Nota: Fuente propia de la investigación. 
cuantificar la concentración y los valores ambientales, dentro de la industria gráfica.

Se identificaron un total de 16 AAs y 5 IAs, su evaluación se realizó mediante el uso de la matriz MIIA y por la definición de criterios de campo para los distintos factores de la matriz, con la finalidad de eliminar la subjetividad propia de esta metodología y así utilizar datos más atinentes al DP. Los valores y los criterios de evaluación para cada AA fueron validados mediante un grupo focal.

La evaluación, cuantificación y posterior priorización de los AAs determinó que en total existen; cuatro AA con un IA severo positivo, un AA con un IA moderado positivo, un AA con un IA irrelevante positivo, dos AA con IA moderado negativo, cinco AA con IA severo negativo y tres AA con IA critico negativo

Dentro de los AAs con valores de impacto beneficioso al ambiente están: generación de $\mathrm{RS}$ reciclables $(+86)$, generación de RS no reciclables $(+57)$, generación de RS peligrosos (+49), generación de AR con solventes y tintas $(+38)$, generación de RS especiales $(+35)$ y generación de AR especiales $(+33)$. Lo anterior se debe a que los RS y las AR con tintas y solventes se tratan a través de un gestor externo autorizado por el MS y la generación AR especiales tiene un caudal de salida bajo $\left(0.25 \pm 0.02 \mathrm{~m}^{3} / \mathrm{h}\right)$.

Los tres AAs con valores de impacto más negativo al medio ambiente son: emisión de VOCs (-81), luminosidad (-77) y consumo de papel (-75). Esto se debe a que los valores medidos sobrepasan los VLAED para VOCs y son bajos según la Norma INTE-31-08-06-00 para el AA de luminosidad. En el caso del AA "consumo de papel", en el DP se utiliza en toda la institución y en alta cantidad (1993,5 kg/mes), lo que afecta los factores de intensidad y extensión, lo que aumenta el valor final en la MIIA. Por otra parte, los principales valores promedios de los IAs de carácter negativo son: contaminación de la atmósfera, afectación a la salud y agotamiento de recursos no renovables, los cuales coinciden con los respectivos AAs.

El proceso de evaluación de impacto ambiental es importante porque permite a la industria autogestionarse para generar medidas preventivas, correctivas y compensatorias, con actividades y plazos definidos para mejorar su gestión en pro del ambiente, y con ello mejorar su imagen empresarial ante la sociedad.

\section{DECLARACIÓN DE LA CONTRIBUCIÓN DE LOS AUTORES}

El porcentaje total de contribución para la conceptualización, preparación y corrección de este artículo fue: W.V.G. 25 $\%$, J.S.B. $25 \%$, J.M.B $25 \%$ y B.A.G. $25 \%$.

\section{DECLARACIÓN DE DISPO- NIBILIDAD DE LOS DATOS}

Los datos que respaldan los resultados de este estudio serán puestos a disposición por el autor correspondiente [W.V.G], previa solicitud razonable.

\section{REFERENCIAS}

Corporación Financiera Internacional. (2007). Guía sobre medio ambiente, salud y seguridad para la industria gráfica. Grupo del Banco Mundial, México. Recuperado de https://www.ifc.org/wps/wcm/connect/15fa498d-5d78-4b75-8a98-743467ed78 b 9/ 0000199659 E S es\%2 B P oultry $\% 2 \mathrm{BProduction}-\% 2 \mathrm{Brev} \% 2 \mathrm{Bcc}$. pdf?MOD=AJPERES\&CVID=jkD2Fyp

Dimate, J. \& Chirley, N. (2014). Evaluación de los impactos ambientales generados por el uso del papel en la impresión de billetes de lotería. Universidad Libre de Bogotá, Colombia. 
Ding, L.; Chen, Y \& Fan, J. (2011). An overview of the treatment of print ink wastewaters. Journal of Environmental Chemistry and Ecotoxicology, 3(10), 272-276.

García, C.; Leone, I., \& Williams, E. (2014). Buenas Prácticas Ambientales en Industrias Gráficas y Afines; Unión Gráfica Argentina Regional, Rosario, Argentina, 11-31.

Instituto Nacional de Seguridad, Salud y Bienestar en el Trabajo (INSSBT). (2019). Limites de exposición profesional para agentes quimicos en España. INSSBT, Madrid: España. Recuperado de http://bdlep.inssbt.es/LEP/

Medaglia, C., \& Vargas, F. (2013). Oportunidades para la industria gráfica costarricense en $\mathrm{Pa}$ namá. PROCOMER, San José, Costa Rica.

Ministerio de Ambiente y Energía. (2013). Decreto 37788-S-MINAE "Reglamento General para la Clasificación y Manejo de Residuos Peligrosos". San José, Costa Rica. http://www.pgrweb.go.cr/scij/Busqueda/ Normativa/Normas/nrm_texto_completo. aspx?nValor1=1\&nValor2=75279\#ddown

Ministerio de Ambiente y Energía \& Ministerio de Salud. (2006). Decreto 32966 “Guía Estudios de Impacto Ambiental y Pronósticos-Plan de Gestión Ambiental, valoración de los impactos ambientales y términos de referencia”. San José, Costa Rica. Recuperado de http://obturcaribe. ucr.ac.cr/documentos-publicaciones/legislacion/ ambiental/150-decreto-32966-manual-de-eia/file

Ministerio de Medio Ambiente. (2013). Manual de Buenas Prácticas Ambientales en la Familia Profesional: Industrias Gráficas. España.

Mora, J.; Molina, O. \& Sibaja, J. (2016). Aplicación de un método para evaluar el impacto ambiental de proyectos de construcción de edificaciones universitarias. Tecnología en Marcha,
29(3), 132-145. https://doi.org/10.18845/ tm.v29i3.2893

Occupational Safety and Health Branch Labour Department. (2004). Chemical Safety in the Workplace: Guidance Notes on Chemical Safety in Printing Industry. Recuperado de https://www.labour.gov.hk/eng/public/os/C/ GN_Printing.pdf

Prica, M.; Kecie, V.; Adamovié, S.; Radonié, J. \& Turk, M. (2016). Occupational Exposure to Hazardous Substances in Printing Industry. Springer, 13. 955-972

Stellman, J. (1998). D, Industria de las artes gráficas, fotografía y reproducción. En: D, Richardson (Ed) Enciclopedia de Salud y Seguridad en el Trabajo. (pp.85.2-85.14). Recuperado de: https://www.insst.es/documents/94886/161971/ Sumario+del+Volumen+III/657d0608-26d1-4 ab6-9491-33a78383b1ac

Stellman, J. (2019). Ruido. En: A. Suter. (Ed). Enciclopedia de salud y seguridad en el Trabajo. (pp.47.1-47.20) Recuperado de https:/www. cso.go.cr/temas_de_interes/higiene/agentes/ fisico/01_ruido_lugar_trabajo.pdf

Torresano, J. (2017). Diseño de una planta de tratamiento de los efluentes líquidos de la industria gráfica. Escuela Politécnica Nacional de Ecuador.

U.S. Environmental Protection Agency. (1998). Método de Referencia del Código de Regulación Federal (CFR) 40-Protection of Environment, Apéndice B de la Parte 50. National Technical Information Service http://www. epa.gov/ttn/catc/products.html\#aptecrpts

U.S. Environmental Protection Agency. (1999). Método TO-17 "Determination of Volatile Organic Compounds in Ambient Air Using Active Sampling Onto Sorbent Tubes". National Technical Information Service http://www.epa. gov/ttn/catc/products.html\#aptecrpts

\section{(c) $(1) \Theta$}

Evaluación del impacto ambiental en una industria gráfica, que utiliza impresión litográfica tipo “offset” (Wendy Villalobos-González • José Pablo Sibaja-Brenes • José Carlos Mora-Barrantes • Benjamín Álvarez-Garay) Uniciencia is protected by Attribution-NonCommercial-NoDerivs 3.0 Unported (CC BY-NC-ND 3.0) 